\title{
Niobium superconducting rf cavity fabrication by electrohydraulic forming
}

\author{
E. Cantergiani, ' S. Atieh, F. Léaux, A. T. Perez Fontenla, S. Prunet, L. Dufay-Chanat, T. Koettig, \\ F. Bertinelli, O. Capatina, G. Favre, and F. Gerigk \\ CERN, CH-1211, Geneva 23, Switzerland
}

\author{
A. C. Jeanson, J. Fuzeau, G. Avrillaud, D. Alleman, J. Bonafe, and P. Marty \\ Bmax, Z.I. Thibaud 30 Bd de Thibaud, 31104 Toulouse, France
}

(Received 24 June 2016; published 17 November 2016)

\begin{abstract}
Superconducting rf (SRF) cavities are traditionally fabricated from superconducting material sheets or made of copper coated with superconducting material, followed by trim machining and electron-beam welding. An alternative technique to traditional shaping methods, such as deep-drawing and spinning, is electrohydraulic forming (EHF). In EHF, half-cells are obtained through ultrahigh-speed deformation of blank sheets, using shockwaves induced in water by a pulsed electrical discharge. With respect to traditional methods, such a highly dynamic process can yield interesting results in terms of effectiveness, repeatability, final shape precision, higher formability, and reduced springback. In this paper, the first results of EHF on high purity niobium are presented and discussed. The simulations performed in order to master the multiphysics phenomena of EHF and to adjust its process parameters are presented. The microstructures of niobium halfcells produced by EHF and by spinning have been compared in terms of damage created in the material during the forming operation. The damage was assessed through hardness measurements, residual resistivity ratio (RRR) measurements, and electron backscattered diffraction analyses. It was found that EHF does not worsen the damage of the material during forming and instead, some areas of the half-cell have shown lower damage compared to spinning. Moreover, EHF is particularly advantageous to reduce the forming time, preserve roughness, and to meet the final required shape accuracy.
\end{abstract}

DOI: 10.1103/PhysRevAccelBeams.19.114703

\section{INTRODUCTION}

Electrohydraulic formed cavities are of interest for several SRF applications at CERN. The technique can be used to produce copper cavities, which are then coated with a thin niobium layer, or to form bulk niobium cavities. The construction of the Future Circular Collider (FCC) requires the use of several superconducting $\mathrm{rf}(\mathrm{SRF})$ beta $=$ 1 elliptical cavities [1]. Depending on the physics goals of FCC, these cavities are expected to have between 1 and 5 cells and to operate at frequencies of 700 or $800 \mathrm{MHz}$. A frequency of $400 \mathrm{MHz}$ is used in the presently operating Large Hadron Collider (LHC), where the use of electrohydraulic forming (EHF) is explored for the production of new spare cavities. The first EHF cavity shapes at CERN have been made in the frame of the $704 \mathrm{MHz}$ HighGradient R\&D program [2], where 5-cell bulk niobium cavities are expected to reach accelerating gradients of $25 \mathrm{MV} / \mathrm{m}$ at $Q$ values of $1 \times 10^{10}$. In the past decades, several forming techniques such as deep-drawing,

\footnotetext{
*Corresponding author. Elisa.Cantergiani@bmax.com
}

Published by the American Physical Society under the terms of the Creative Commons Attribution 3.0 License. Further distribution of this work must maintain attribution to the author(s) and the published article's title, journal citation, and DOI. spinning, and hydroforming were investigated to produce elliptical half-cells. Spinning is currently used at CERN to produce half-cells of copper which undergo electron-beam welding at the equator in order to obtain a full cavity [3]. At DESY, hydroforming was implemented to produce full cells by starting from seamless niobium tubes [4-5]. All these traditional forming processes present several drawbacks; in particular, deep-drawing cannot produce complex shapes with high accuracy and according to literature [6] it requires high-tonnage hydraulic presses when shaping large half-cells. Furthermore, for the production of SRF half-cells, a separate coining step is necessary to obtain the curvature at the iris. Moreover, deep-drawing is particularly affected by springback which can result in high deviation from the nominal geometry causing fluctuations in the resonating frequency [7-8]. For large components, spinning is preferred; however, it requires multiple forming steps and the optimization of several parameters including feed ratio, roller path, roller design, and spinning ratio to be effective [9]. At CERN, spinning was successfully used to form half-cells of oxygen-free electronic (OFE) copper but intermediate annealing treatments were necessary during forming operation. These annealing treatments are feasible for copper but not for niobium in order to avoid degradation of its purity. Both deep-drawing and spinning leave a damaged layer on the rf surface of the shaped cavity which needs to be removed by a chemical treatment in order to 
improve the accelerating gradient $\left(E_{\text {acc }}\right)$. According to literature [6], a chemical treatment to remove at least $100-150 \mu \mathrm{m}$ is necessary to clean the rf surface from the damaged layer caused by forming operations.

Concerning hydroforming, its main advantage is the production of a complete cell without the critical equator welds; however it requires several forming and calibrations stages and a careful optimization of the forming parameters. Hydroforming produces surfaces which are rougher than the one obtained by deep-drawing and it still requires the removal of 100-200 $\mu \mathrm{m}$ by buffered chemical polishing [5]. Another drawback of hydroforming is that only the outside shape of the cell is formed with precision, while the accuracy of the rf surface highly depends on the thickness and tolerance of the starting tube [5]. This requires a high control of the dimensions of the initial tube and of the hydroforming parameters [5].

Starting from this background, it is evident that a forming process which could ensure high shape accuracy, high reproducibility and in the same time limit the damage left in the material and the time required for forming would be preferable. High strain rate forming processes such as magnetic pulse forming and electrohydraulic forming (EHF) could be suitable for the production of SRF cavities. According to literature $[10,11]$, high-speed forming techniques are particularly advantageous to limit springback and to increase the formability of metals. From an industrial point of view they are attractive for time and cost savings given that the whole forming process can be done in few milliseconds and that no punch is necessary [12]. The high shape accuracy that can be obtained by EHF of niobium SRF half-cells was already proven by Atieh et al. [8], who reported an accuracy of $\pm 200 \mu \mathrm{m}$ compared to $\pm 600 \mu \mathrm{m}$ of traditional forming processes. In the same work, Atieh et al. [8] have shown that EHF preserves the initial surface roughness of the starting sheet.

In this paper we present results of EHF of $704 \mathrm{MHz}$ SRF half-cells and compare this high-speed forming technique with spinning in terms of damage left in the material (niobium). The damage in the microstructure is assessed with hardness, residual resistivity ratio measurements and electron backscattered diffraction (EBSD). The microstructure of electro-hydro formed niobium was further investigated by using transmission electron microscope (TEM).

\section{ELECTRO-HYDRAULIC FORMING OF NIOBIUM HALF-CELLS}

\section{A. Description of electrohydraulic forming}

In collaboration with the company Bmax, an activity to form half-cells of high purity niobium $(R R R \geq 300)$ was started. The main components to perform electrohydraulic forming are shown in Fig. 1 and they include a control cabinet, a capacitor bank, and the tank where the forming of the blank sheet takes place. A schematic view of this tank is shown in Fig. 2.

Two electrodes are positioned inside this tank and connected to a capacitor, while vacuum is created between the die and the sheet in order to avoid the formation of defects during the fast forming. The tank is then filled with water. When the high power switch is closed, the electrical energy stored in the capacitor is released between the electrodes creating a nonthermal plasma. This nonthermal plasma creates a fast bubble expansion which will result in a rapid shock wave that propagates in water causing the plastic deformation of the metallic sheet against the die [8-12]. When the sheet is subjected to EHF, the impact velocities range from a minimum of $50 \mathrm{~m} / \mathrm{s}$ up to $200 \mathrm{~m} / \mathrm{s}$ and the material undergoes strain rates in the order of $10^{3}-10^{4} \mathrm{~s}^{-1}$. According to literature [14], for such high strain rates, the material shows a viscoplastic behavior which may cause an increase in formability. Indeed, high strain rate forming techniques would cause a more uniform dislocation distributions hindering or delaying the formation of dislocation cells [14].

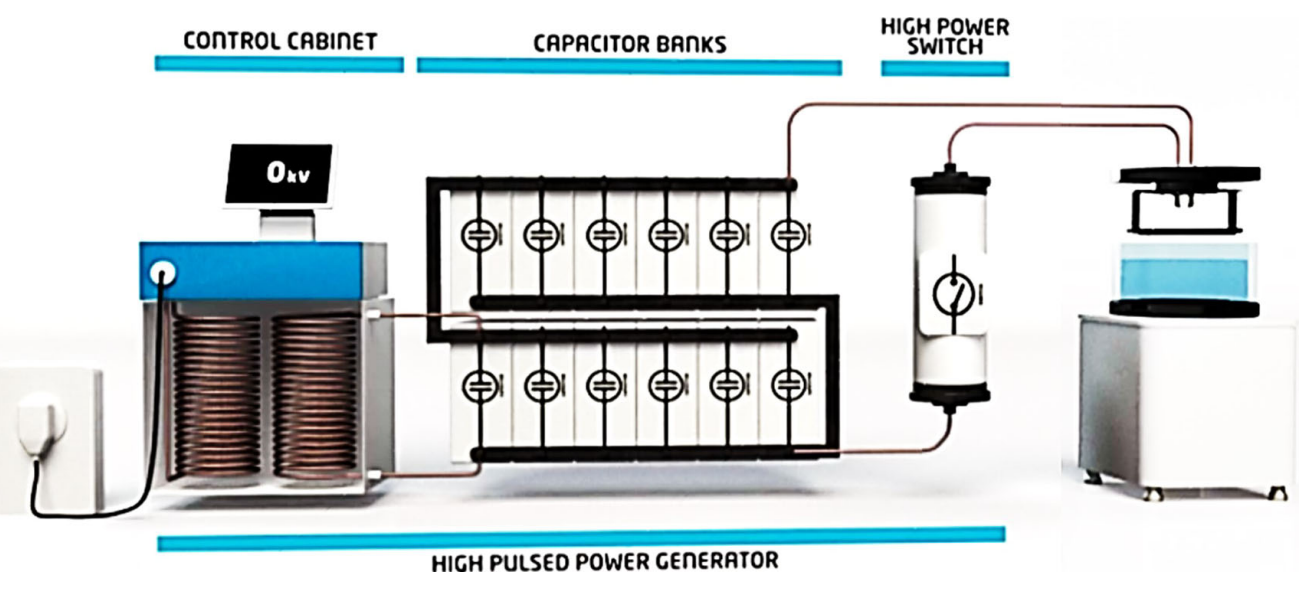

FIG. 1. Equipment used for electrohydraulic forming $[8,13]$. 


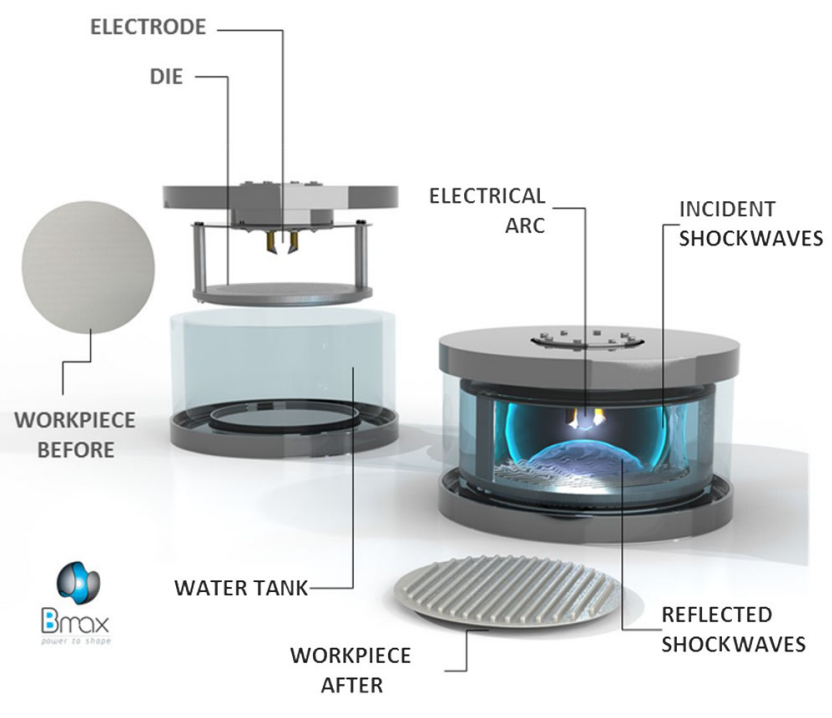

FIG. 2. EHF tank.

\section{B. Numerical simulation of electrohydraulic forming}

\section{Characterization of mechanical properties of niobium at high strain rates}

The main parameters which need to be chosen in order to perform electrohydraulic forming include the position of electrodes, the input energy stored in the capacitor, the number of pulses, and the duration of each pulse. In order to predict the electrical power delivered in the arc during a discharge, Bmax has developed a proprietary model which calculates this power as function of inductance, capacitance, voltage of the generator, and gap between the electrodes. The number of pulses and the duration of each pulse are optimized by performing a finite element simulation using the commercial software LS-DYNA ${ }^{\circledR}$ by taking into account the yield stress and the thickness of the material to be formed. Most metallic materials exhibit mechanical properties which are dependent on the strain rate [15]. In literature [14], it was reported that the yield strength of pure BCC metals (such as niobium) has a strong strain rate dependency, while the strain hardening variation as function of strain rate is negligible. Thus, for enhanced predictability of EHF simulations, it is fundamental to characterize the evolution of the mechanical properties of niobium as function of strain rate by using a suitable constitutive model. The evolution of mechanical properties as function of strain rate are taken into account during EHF simulations. Several viscoplastic constitutive models have been proposed to describe the mechanical behavior of metals at high strain rates; some of them are purely empirical as the Johnson-Cook (JC) model [16], while others, as the Zerilli-Armstrong (ZA) model, are based on dislocations mechanics [17]. In order to obtain the constitutive behavior for the niobium used for the production of SRF half-cells, Bmax has developed a setup to characterize the material by electromagnetic expansion. The experimental setup used for the characterization of niobium sheets at high strain rates is similar to what has been described for tube expansion tests [18] and for ring expansion tests [19]. A schematic view of a tube expansion test is shown in Fig. 3. A coil is used as an inductor and it is connected to a generator. The coil is crossed by a rapid discharge of current (measured through a Rogowski coil) which produces a magnetic field. This magnetic field will induce current in the metallic piece whose mechanical properties need to be characterized. The interaction between the magnetic field created by the inductor and the electrical current generated in the piece produces Lorentz forces which will expand at high speed the material to be analyzed. During the expansion test, the speed of deformation is measured by using a photon doppler velocimeter (PDV).

Once the speed of deformation of the material has been obtained experimentally, theoretical simulations of an electromagnetic expansion test are performed by using the commercial software LS-DYNA [21]. The input of these simulations includes the initial geometry of the metallic specimen, the electrical current crossing the inductor, and the constitutive behavior of the material. During these simulations, the material parameters of the constitutive behavior are optimized by an iterative procedure in order to minimize the mean-square error between the theoretical and the experimental speed of deformation. The iterative procedure of optimization is done using the commercial tool LS-Opt ${ }^{\circledR}$ [18]. An example of fitting of the experimental velocity of expansion is shown in Fig. 4.

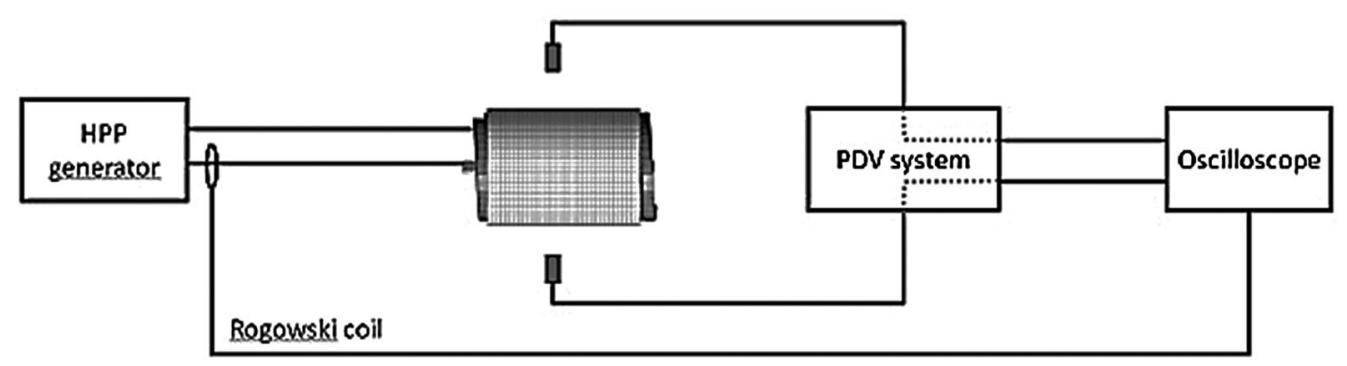

FIG. 3. Setup of expansion test for characterization of metals at high strain rates [20]. 


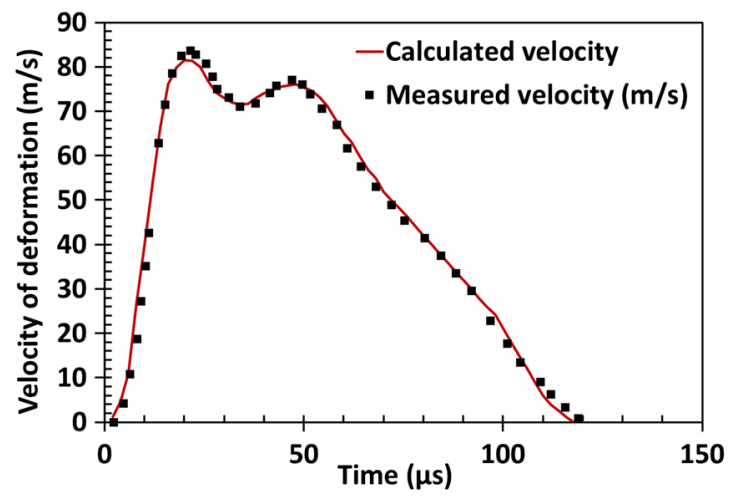

FIG. 4. Experimental and simulated velocity versus time curves for tube expansion test.

According to results obtained by the electromagnetic expansion tests, the behavior of niobium can be described by using the Zerilli-Armstrong model expressed by Eq. (1):

$\sigma=C_{1}+C_{2} \times \exp \left[\left(-C_{3}+C_{4} \ln \left(\dot{\varepsilon} / \dot{\varepsilon}_{0}\right)\right) T\right]+K \varepsilon_{p}^{n}$

where, $\varepsilon_{p}$ is the plastic strain, $\dot{\varepsilon}$ is the strain rate, $\dot{\varepsilon}_{0}$ is the reference strain rate (set to $1 \mathrm{~s}^{-1}$ ), $T$ represents the current temperature of $290 \mathrm{~K}, n$ the strain hardening exponent, while $C_{1}, C_{2}, C_{3}, C_{4}$ and $K$ are all material constants. By fitting the results of five electromagnetic expansion tests performed on specimens of niobium, the values of the constants were found. The results have confirmed a high sensitivity of mechanical properties of niobium to strain rate.

\section{Simulation of electrohydraulic forming of SRF niobium half-cells}

EHF uses the pressure waves generated in the water to form the metal sheet. This pressure is generated by a high and fast pulse of current going through either the water itself or a small conducting wire connecting the two electrodes in the water. The evolution of this electrical power over time is an input for the forming simulations and it is calculated by a proprietary model depending on the electrical parameters of the generator. Successively, in a 2D axisymmetric EHF model done with LS-DYNA, this calculated power is used to generate pressure waves in an Eulerian mesh representing the water. At each time step, this mesh is coupled to the Langrangian mesh representing the metal blank, so that the pressure generated in the water is transferred as a force in the sheet to be formed. After a forming shot is finished, the partially formed part with its residual stress and strain distribution is used as a starting blank to simulate the next forming shot.

For the forming of half-cells in niobium, the complete EHF forming operation was done in 13 pulses with the

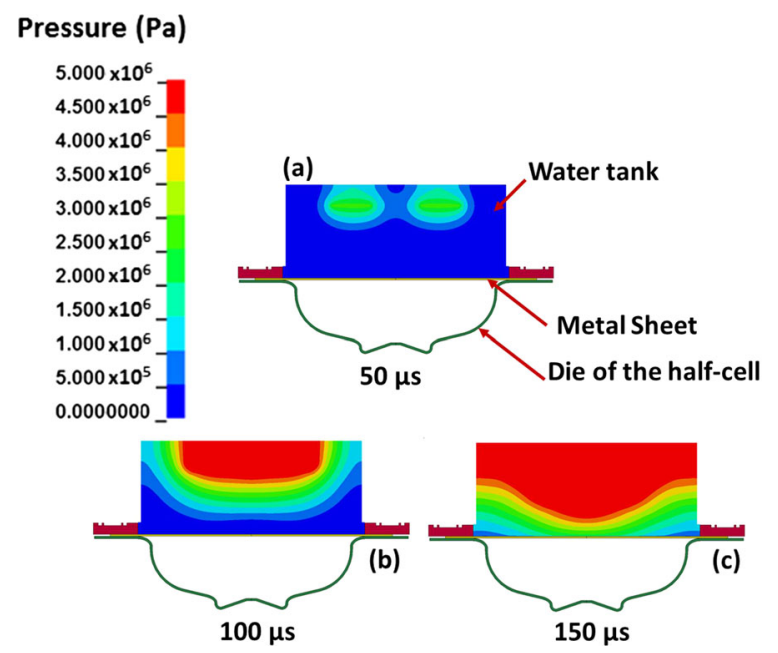

FIG. 5. Pressure evolution inside the water tank after the electrical discharge between the electrodes: after $50 \mu \mathrm{s}$ from the discharge (a), after $100 \mu \mathrm{s}$ (b), and after $150 \mu \mathrm{s}$ (c).

current pulse between the electrodes lasting for $300 \mu \mathrm{s}$, while the total operation time for one shot from the discharge of the current pulse to the equilibrium inside the chamber was $200 \mathrm{~ms}$. The pressure evolution inside the water chamber before starting the deformation of the sheet is shown in Figs. 5(a), 5(b), and 5(c) at different times, while the evolution of the sheet forming during the different shots is shown in Fig. 6.

From these simulations, the plastic strain distribution along the profile of the half-cell can be extracted in order to highlight the zones where strain concentration might become critical enough to cause tearing or failure. An example of the strain distribution obtained along the profile for the niobium half-cell is shown in Fig. 7. A strain concentration is expected at the curvature at the bottom; however, the strain level is not high enough to cause failure and it is out of the iris zone which is important for the halfcell functionality.

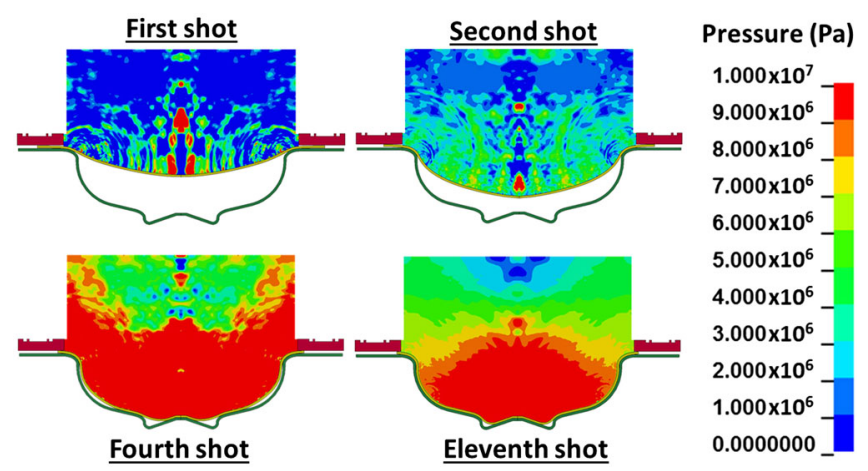

FIG. 6. Pressure evolution inside the water tank for different shots during forming of niobium. 


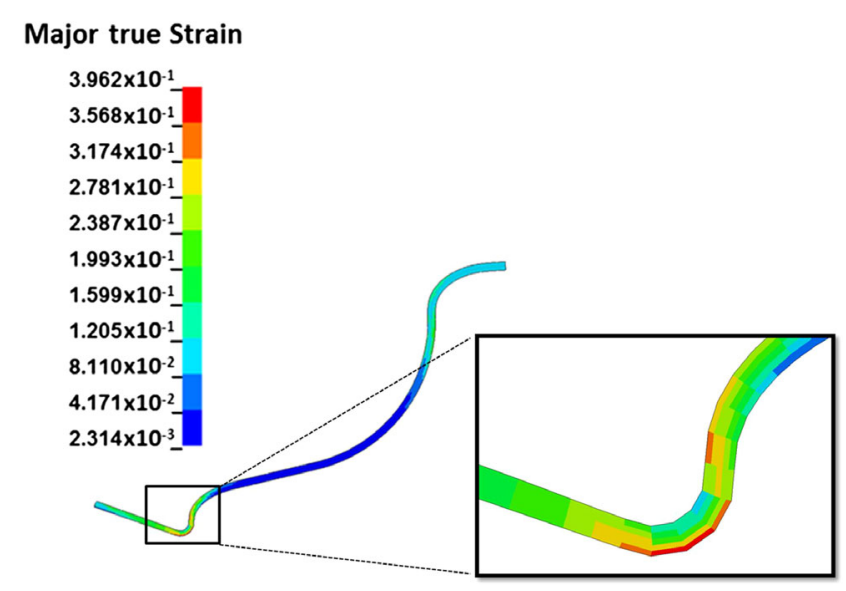

FIG. 7. Strain distribution through thickness after EHF of a niobium half-cell.

\section{RESULTS}

\section{A. Characterization of niobium microstructure before forming}

The material used for EHF was high purity niobium (RRR $\geq 300)$ supplied by Ningxia Orient Tantalum Industry Co., while niobium with the same characteristics but supplied by Plansee was used to form a $704 \mathrm{MHz}$ halfcell by traditional spinning process. RRR values, Vickers hardness (HV10) on the surface, and Vickers microhardness (HV0.2) through thickness were measured on niobium from both suppliers. All the values are summarized in Table I and they comply with CERN requirements for the production of SRF cavities.

RRR was calculated as the ratio between the resistivity of niobium at room temperature $(300 \mathrm{~K})$ and the resistivity of niobium at liquid helium temperature $(4.2 \mathrm{~K})$ at standard atmospheric pressure [22]. Since the transition of niobium from the normal conducting state to the superconducting state occurs at $9.3 \mathrm{~K}$, the method used in this work to determine the RRR value was based on the dc method and successive extraction of the resistivity at $4.2 \mathrm{~K}$ by extrapolation of the curve of resistivity versus temperature as suggested in literature [23-24].

The niobium used at CERN for the production of SRF cavities must have a RRR value above 300 . RRR specimens were cut as slices of $2 \mathrm{~mm} \times 2 \mathrm{~mm}$ of cross section and length of $100 \mathrm{~mm}$ by using electro discharge machining

TABLE I. Hardness, RRR, and grain size of niobium material used for electrohydraulic forming (Ningxia) and for spinning (Plansee).

\begin{tabular}{lcccc}
\hline \hline $\begin{array}{l}\mathrm{Nb} \\
\text { supplier }\end{array}$ & HV 10 & $\begin{array}{c}\text { HV 0.2 } \\
\text { (through thickness) }\end{array}$ & RRR & $\begin{array}{c}\text { Grain } \\
\text { size }\end{array}$ \\
\hline Ningxia & 51 & 57 & 382 & $53 \mu \mathrm{m}$ \\
Plansee & 47 & 62 & 340 & $63 \mu \mathrm{m}$ \\
\hline \hline
\end{tabular}

since it was found that this technique is the less invasive for cutting of niobium [25]. Afterwards, the specimens were degreased and electropolished to remove $150-200 \mu \mathrm{m}$ to ensure complete removal of the oxidation caused by machining.

The microstructure was analyzed to verify that the initial sheet had equiaxed grains and uniform grain size distribution. In Fig. 8(a) the microstructure of the niobium surface is shown, while its microstructure through thickness for both suppliers is shown in Figs. 8(b) and 8(c). Tensile tests at a strain rate of $10^{-3} \mathrm{~s}^{-1}$ were performed on niobium from both suppliers. Both starting sheets show comparable mechanical properties; in particular, the yield stress was $56 \pm 2 \mathrm{MPa}$ for Plansee and $67 \pm 5 \mathrm{MPa}$ for Ningxia, while the ultimate tensile strength was $177 \pm$ 2.6 MPa for Plansee and $171 \pm 1.5 \mathrm{MPa}$ for Ningxia. The strain hardening exponent was obtained from true stress strain curves and the value of 0.35 was found for Plansee and the value of 0.31 was obtained for Ningxia.

Detailed investigations were then undertaken to compare the damage caused by the two forming techniques inside the material.

\section{B. Characterization of niobium microstructure after forming}

\section{Hardness measurements}

After forming, the half-cell obtained by spinning needed to be compared with the half-cell formed by EHF in terms of damage left in the material. The damage caused during forming is related to an increase in density of dislocations. For this reason, a straightforward parameter that can be measured to evaluate the damage of the material is hardness. As a consequence of the increase in dislocations density during forming, hardness is expected to increase compared to the nonformed blank. Thus, specimens of $15 \times 15 \mathrm{~mm}$ were cut along the profile of the half-cell formed by spinning and along the profile of the half-cell formed by EHF as shown in Fig. 9.

Vickers hardness was measured on both internal and external surfaces by applying a load of $10 \mathrm{~kg}$ for $15 \mathrm{sec}$ (HV 10). During spinning, the internal surface, which is defined as the rf surface, is in contact with the mandrel, while the external surface is shaped by the external roller. In EHF the internal surface is in contact with water shockwaves, while the external surfaces impacts against the die. In Fig. 10 the results of Vickers hardness (HV 10) are shown for both forming techniques while the hardness of the not-formed blank is reported for reference. The position of the specimens along the profile was numbered so that specimen 5 is located at the equator and specimen 15 at the iris. Each point shown in Fig. 10 is the average of at least 12 measurements. The error bars associated with each set of measurements are reported only for the internal surfaces for clarity, but similar variation was obtained for external surfaces. 

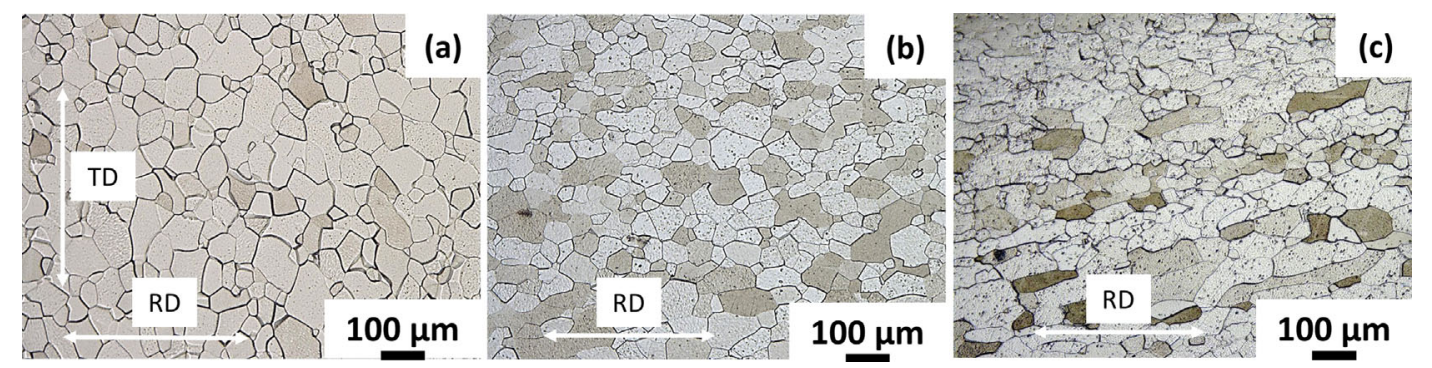

FIG. 8. Microstructure of Niobium (Ningxia) used for EHF of half-cells: (a) surface of the metal sheet (RD indicates the rolling direction and TD the transversal direction), (b) through thickness microstructure (Ningxia), (c) through thickness microstructure (Plansee).

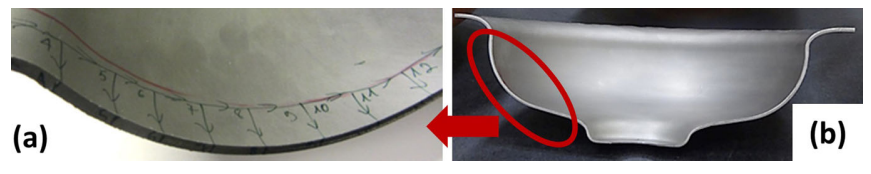

FIG. 9. Images of EHF niobium: (a) example of specimens which were extracted along the profile of the half-cell; (b) section of a half-cell.

From Fig. 10 one can see that both forming technologies increase the hardness of the material; however, spinning shows a higher increase of hardness compared to EHF starting from the center of the half-cell profile (position 9) up to the equator (position 5). At the iris instead, the two technologies cause the same change in hardness. This hardness trend can be related directly to the forming process. Indeed, during EHF, the most critical part to be formed is the iris and more pulses are necessary to shape the material in this zone. On the other hand, for spinning, the most critical part to be formed is at the equator, where the external roller has to perform several passes to shape the blank. From this graph, by comparing the curves of the internal surfaces (rf surfaces) for the two forming technologies, it is clear that spinning causes higher hardness compared to electrohydraulic forming, especially

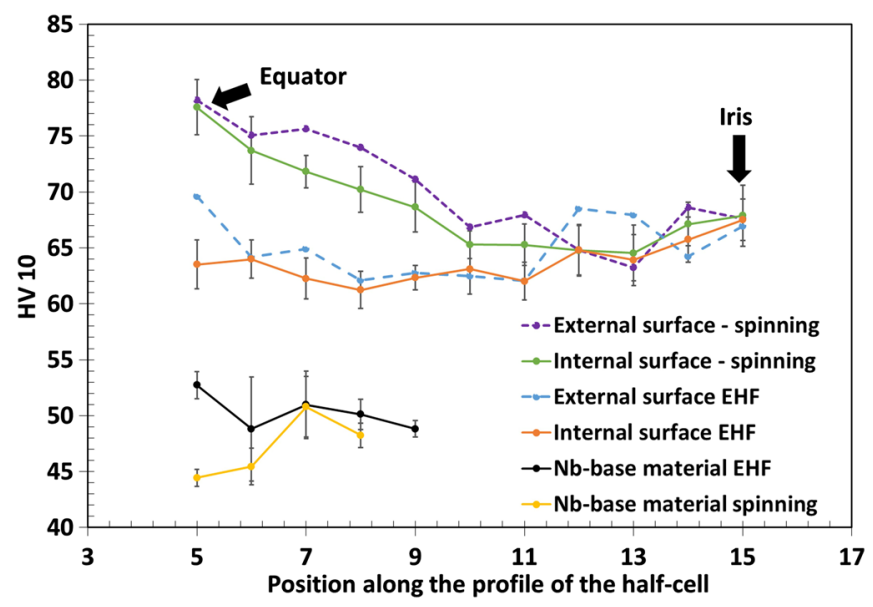

FIG. 10. Vickers hardness (HV 10) measured on the external and internal surfaces for half-cells formed by spinning and EHF. at the equator. Moreover, for the half-cell obtained by spinning, the hardness of external and internal surface has the same trend; while for EHF at the equator, the hardness of the external surface is higher than the one of the internal surface.

Vickers microhardness (HV 0.2) through thickness was measured for both forming techniques after polishing of the specimens up to mirror finish. The average hardness through thickness of the half-cell obtained by spinning exceeds the hardness of the half-cell formed by EHF.

\section{RRR measurements}

According to literature [26], lattice defects (such as dislocations) interfere with electron conduction; in particular, if the amount of impurities and lattice defects present in the material is increased, the residual resistivity ratio will decrease [22]. For this reason, RRR values are expected to decrease after forming as a consequence of the increase in density of dislocations. RRR specimens were extracted both in radial and circumferential direction for half-cells formed by spinning and EHF. The values of RRR measured for the two series of specimens from both technologies were then compared. A dedicated sample holder was designed to avoid a change of curvature of the specimens. Figure 11(a) shows zones of a half-cell where specimens were cut, while Fig. 11(b) shows the holder that was used for the RRR test. Six specimens were tested for each zone.

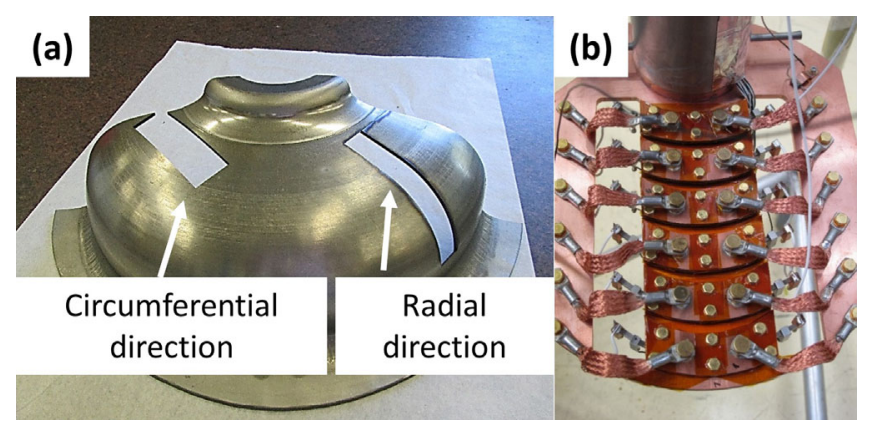

FIG. 11. (a) Half-cell of niobium showing circumferential and radial zones of cutting of RRR specimens, (b) sample holder used for RRR specimens. 


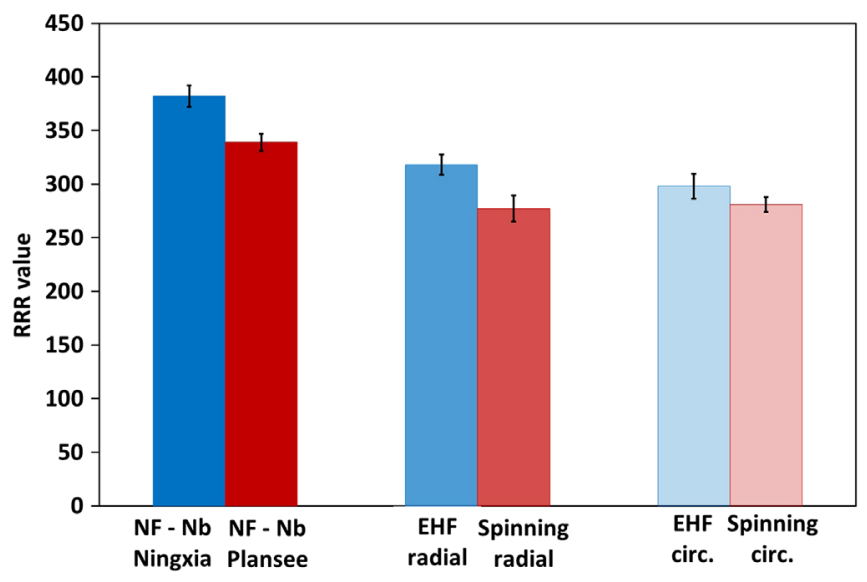

FIG. 12. RRR values obtained for nonformed niobium (NF- Nb) and for half-cells formed by EHF or spinning. "Circ." indicates specimens which were extracted circumferentially close to the iris.

The average RRR values and the corresponding standard deviation are reported in Fig. 12.

As shown in Fig. 12, both forming techniques cause a decrease of RRR values. The average drop of RRR values in the radial direction is the same for both forming techniques: 64 for EHF against 62 for spinning. For specimens extracted in the circumferential direction close to the iris, the average drop of RRR after EHF is 84 against 60 for spinning. This higher drop of RRR found for EHF corresponds to the location of the two peaks of Vickers hardness that were recorded on the external surface for specimens extracted from the half-cell obtained by EHF (points in position 12 and 13 in Fig. 10). Due to this higher drop of RRR value compared to spinning, the RRR specimens extracted in the circumferential direction at the iris of the EHF half-cell were subjected to a heat treatment to recover dislocations and obtain an improvement of RRR back to the value of the not-formed niobium. One set of samples was annealed at $600^{\circ} \mathrm{C}-5 \mathrm{~h}$, a second set of samples was annealed at $700{ }^{\circ} \mathrm{C}-5 \mathrm{~h}$, and another set at $800^{\circ} \mathrm{C}-5 \mathrm{~h}$. Each heat treatment was performed in vacuum $\left(5 \times 10^{-6} \mathrm{mbar}\right)$. The range of temperature, the annealing time, and the level of vacuum were chosen considering the parameters reported in literature for hydrogen degassing of niobium [27].

After the heat treatment, the specimens were degreased and RRR values were measured again. Figure 13 shows the evolution of RRR values as a function of temperature. The microstructure of the specimens was then analyzed in terms of Vickers hardness (HV 0.2) and grain size to prove that no recrystallization occurred. The values of RRR and Vickers hardness are summarized in Table II.

The RRR value of specimens formed by EHF is fully recovered after heat treatment at $600{ }^{\circ} \mathrm{C}$ for $5 \mathrm{~h}$. A further increase in temperature above $600{ }^{\circ} \mathrm{C}$ does not improve further the RRR values. Moreover, a negligible variation of

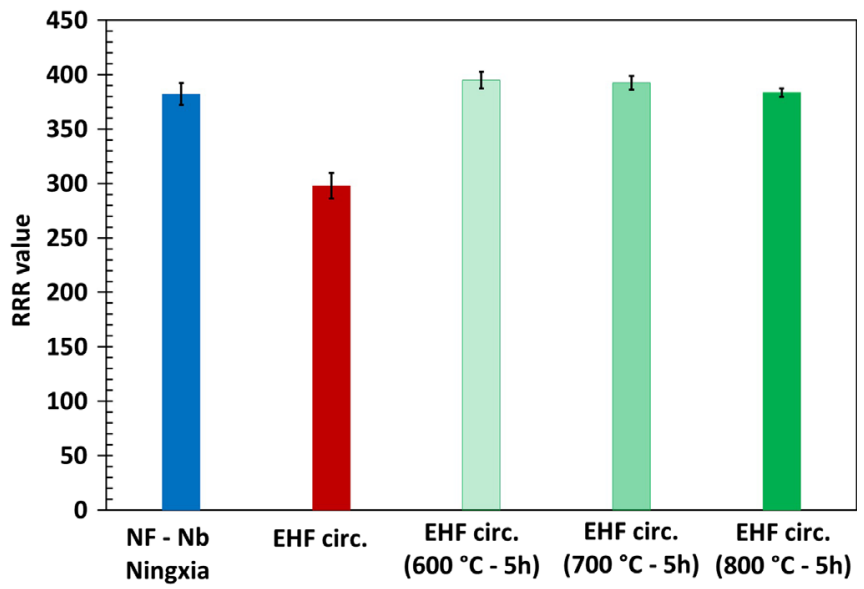

FIG. 13. RRR values after heat treatment at different temperatures for $5 \mathrm{~h}$ for specimens obtained in circumferential direction (circ.) for a half-cell formed by EHF.

hardness was detected, meaning that the improvement of RRR is only due to recovery of dislocations but no recrystallization took place. As reported in literature [28-29], when recrystallization occurs, new grains are nucleated and they grow causing a decrease in mechanical properties (for example a decrease in hardness). Temperatures above $800^{\circ} \mathrm{C}$ were not tested to avoid recrystallization of niobium.

\section{Electron backscattered diffraction analyses}

Electron backscatter diffraction can be used to assess the level of plastic strain left on a deformed material. The formation of dislocations inside the material causes a variation in lattice orientation and a degradation of the diffraction pattern [30]. In this work, the through thickness strain induced by EHF and spinning was compared by mapping the local misorientation gradient via the so called kernel average misorientation (KAM) criterion. The KAM is calculated as the averaged misorientation between one pixel and its neighboring pixels included in a kernel whose dimensions are defined by the user. In this work, the kernel was a $3 \times 3$, which means that the misorientation of each pixel was compared with the misorientation of pixels included in a $3 \times 3$ square grid as shown in Fig. 14(a).

TABLE II. Values of RRR and Vickers Hardness for specimens of niobium extracted from circumferential direction of EHF half-cell.

\begin{tabular}{lcc}
\hline \hline Sample & RRR & $\begin{array}{c}\text { Vickers hardness } \\
\text { (HV 0.2) }\end{array}$ \\
\hline NF-Nb Ningxia & 382 & 57 \\
EHF_circumferential & 298 & 74 \\
EHF circumferential $\left(600^{\circ} \mathrm{C}-5 \mathrm{~h}\right)$ & 395 & 77.6 \\
EHF circumferential $\left(700^{\circ} \mathrm{C}-5 \mathrm{~h}\right)$ & 392.5 & 74 \\
EHF circumferential $\left(800^{\circ} \mathrm{C}-5 \mathrm{~h}\right)$ & 383.5 & 69 \\
\hline \hline
\end{tabular}


(a)

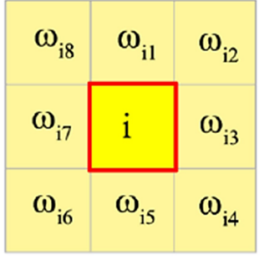

(b)

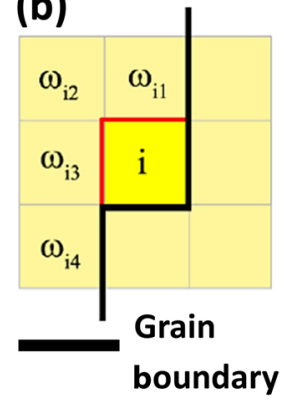

FIG. 14. Close neighbors defined for the calculation of KAM $(3 \times 3)$ in a pixel inside a grain (a) and close to a grain boundary (b) $[31]$.

The misorientation values corresponding to boundaries $\left(>5^{\circ}\right)$ are excluded from the calculations since this is the value which corresponds to low angle grain boundaries Fig. 14(b)[31].

The value of KAM associated with pixel $i$ is calculated as shown in Eq. (2),

$$
\mathrm{KAM}_{i}=\frac{1}{K} \sum_{j=1}^{K} \omega_{i j} \quad \omega_{i j}<\omega_{\text {lim }}
$$

where $K$ is the total number of pixels defined as close neighbors of pixel $i, \omega_{i j}$ is the misorientation angle between pixel $i$ and pixel $j$ and $\omega_{\lim }$ is the misorientation limit (in the current work set to $5^{\circ}$ ) [31]. Once the KAM has been calculated for each pixel, then the average KAM of the whole zone is calculated according to Eq. (3).

$$
\mathrm{KAM}=\frac{1}{N} \sum_{i=1}^{N} \mathrm{KAM}_{i}
$$

where $N$ is the total number of pixels included in an area [31].

As shown by Romanenko and Padamsee [32], local misorientation is induced by the lattice distortion and is directly related to the amount of geometrically necessary dislocations (GNDs) which are produced to accommodate the deformation. According to the work of Romanenko et al. [32] and as reported by Antoine [33], hot spots in SRF cavities are associated with higher misorientation angles and to a higher dislocation density. Moreover, it was reported that during a cold rf test, dislocations can act as preferential sites for precipitation of hydrides and again a higher precipitation of hydrides was responsible for the presence of hot spots in the SRF cavity [32-33].

In the current work, EBSD analyses were performed on specimens extracted at the equator and at the iris for both forming techniques. Specimens were polished down to $0.05 \mu \mathrm{m}$ silica solution and then electropolished to remove $10 \mu \mathrm{m}$ to avoid the presence of a damaged layer left by

(a)

Internal

(b)

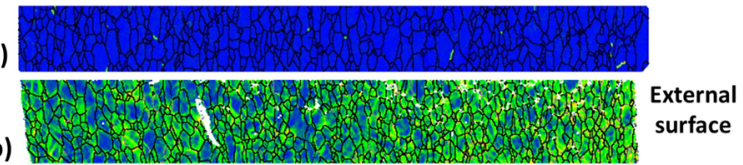

(c)

(d)

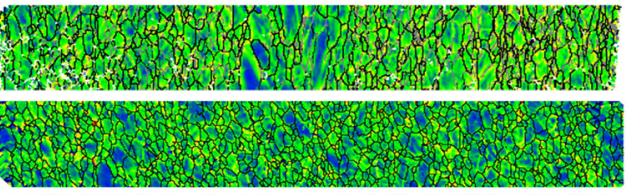

(e)

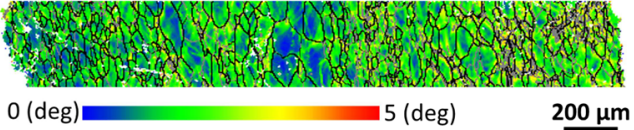

FIG. 15. KAM $(3 \times 3)$ maps for nonformed specimen (a) and for formed specimens extracted from SRF half-cells formed by electrohydraulic forming and by spinning: (b) EHF equator; (c) spinning equator; (d) EHF iris; (e) spinning iris. The external surface of the half-cell is on the right of the image, while the internal surface of the half-cell is on the left of the image. White zones inside the KAM maps represent nonindexed points.

mechanical polishing. EBSD analyses were performed through the whole thickness of the specimens by using a field emission scanning electron microscope Sigma (Zeiss). The microscope was operated at a voltage of $20 \mathrm{kV}$ and a $5 \mu \mathrm{m}$ step was used for the scan through the whole thickness. The KAM values depend on the step size: a big step size, as used in the current work for the study of the whole thickness, allows acquisition of large areas but tends to overestimate the misorientation. For this reason, another series of EBSD analyses was performed with a $1 \mu \mathrm{m}$ step size. However, for this set of analyses, a smaller area $(150 \mu \mathrm{m} \times 200 \mu \mathrm{m})$ was scanned, close to the external and internal surfaces of both specimens extracted from iris and equator for both forming techniques.

Figure 15 shows the EBSD map (KAM $3 \times 3$ as defined in Fig. 14) obtained through the whole thickness for specimens extracted at the equator and the iris for both EHF and spinning. For all the images, the external surface of the half-cell is on the right of the image, while the internal surface of the half-cell is on the left of the image. Since EBSD results are sensitive to experimental conditions, a nonformed specimen of niobium was used as reference. The associated KAM map is shown in Fig. 15(a) and as expected no strain is detected. For a specimen extracted at the equator of the EHF half-cell, a gradient of KAM can be discerned going from the external surface to the internal surface, Fig. 15(b). The internal surface shows lower KAM, due to lower plastic deformation, compared to the external surface, which shows higher plastic deformation. This result is in agreement with the hardness values measured on this sample and presented in Fig. 10 position 5 for EHF. Specimen (c) in Fig. 15, which was extracted at the equator of the half-cell formed by spinning, shows a uniform distribution of KAM through the whole thickness. For specimens extracted at the iris for both forming 

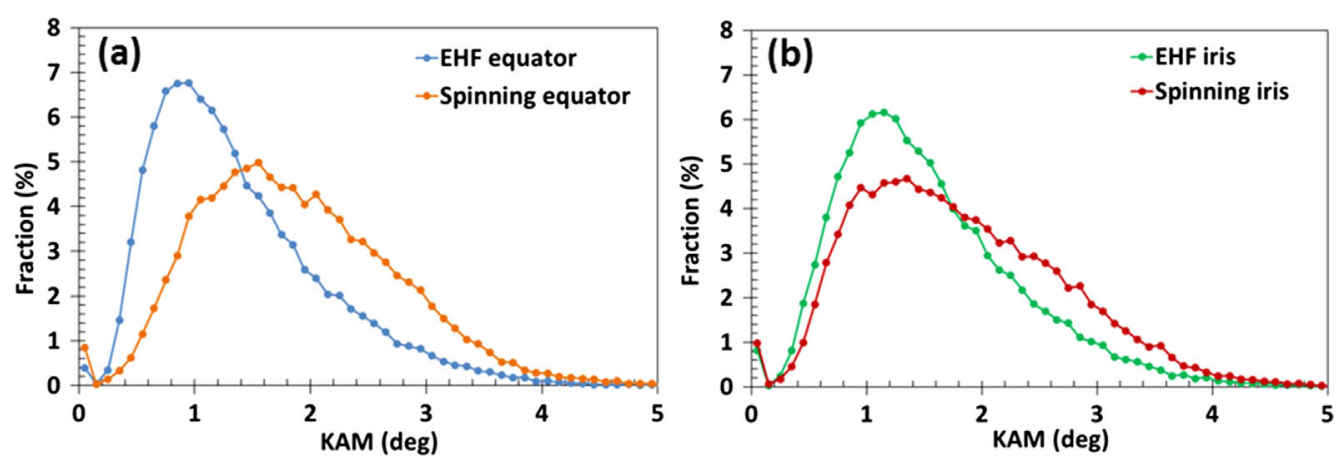

FIG. 16. Local misorientation distribution for specimens extracted from half-cells formed by EHF and spinning: (a) specimens obtained from equator; (b) specimens obtained from iris.

techniques [Figs. 15(d) and 15(e)], the KAM misorientation is uniform through the whole thickness. The KAM distribution of each specimen was extracted for the whole thickness and compared. Results are shown in Fig. 16(a) for specimens extracted at the equator and in Fig. 16(b) for specimens extracted at the iris. From Fig. 16(a) it is noticed that the peak of KAM is shifted toward higher angles for the half-cell formed by spinning compared to the half-cell formed by EHF. This result suggests that, on average through the whole thickness, EHF causes lower misorientation compared to spinning. This is a consequence of a lower misorientation present on the internal surface of the specimen extracted from EHF [Fig. 15(b)] compared to the internal surface of the specimen extracted from spinning [Fig. 15(c)]. On the other hand, at the iris, the local misorientation between the two forming techniques does not differ appreciably [Fig. 16(b)].

Afterwards, another set of EBSD analyses was performed in the first 150-200 $\mu \mathrm{m}$ through thickness close to the external and to the internal surfaces in order to assess more precisely the presence of a damaged layer. The KAM $(3 \times 3)$ maps are shown in Fig. 17, while the distribution of the local misorientation is reported in Fig. 18.

According to the distribution shown in Fig. 18, the two forming techniques seem to cause the same damage at the equator in the first $200 \mu \mathrm{m}$ close to the external surface [Fig. 18(a)], but at the internal surface the damage caused by EHF is lower than the damage encountered for spinning. At the iris [Fig. 18(b)], the misorientation caused by spinning is higher than the one caused by EHF for the external surface; however, the two techniques cause the same level of damage at the internal surface.

\section{Transmission electron microscopy on electrohydraulic formed niobium}

Transmission electron microscopy was used to obtain information about the mechanism of deformation activated during high strain rate forming of niobium. The sample chosen for this investigation was extracted from the equator of an EHF half-cell. This location was chosen to better
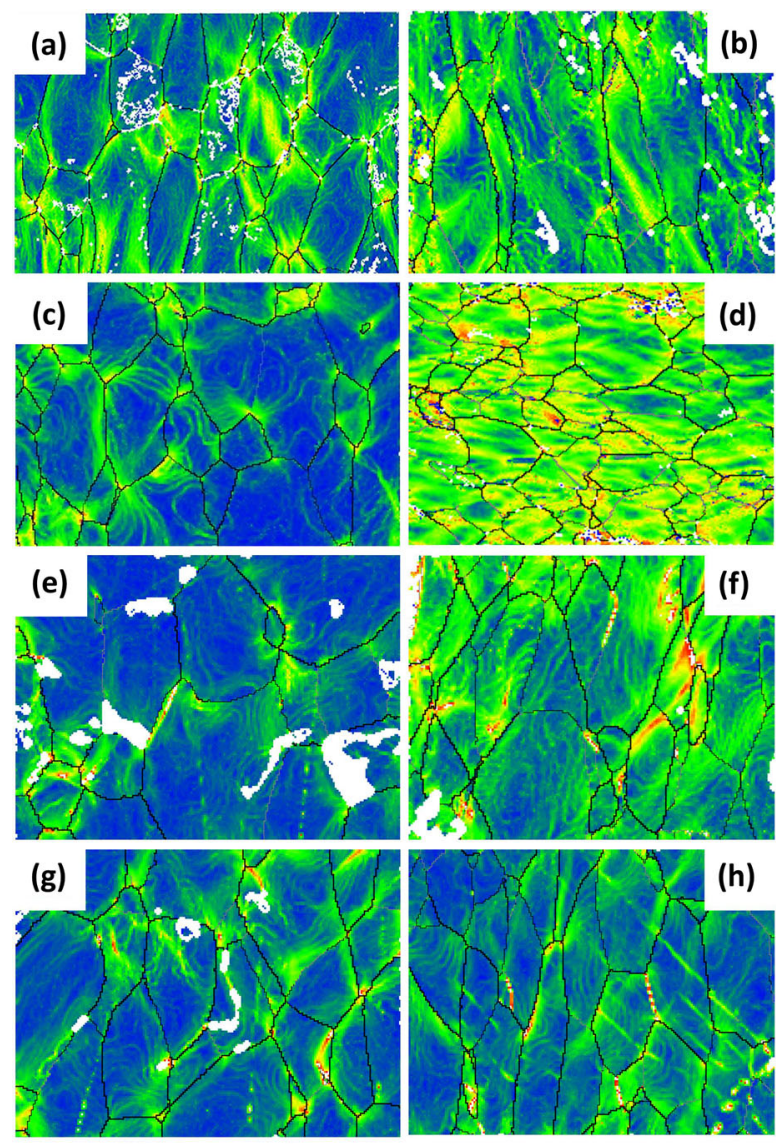

0 (deg)

2 (deg)

$\underline{50 \mu \mathrm{m}}$

FIG. 17. Local misorientation map $($ KAM $3 \times 3)$ through thickness close to external and internal surfaces: (a) external surface equator of EHF half-cell; (b) external surface equator of half-cell formed by spinning; (c) external surface iris of EHF halfcell; (d) external surface iris of half-cell formed by spinning; (e) internal surface equator of EHF half-cell; (f) internal surface equator of half-cell formed by spinning; $(\mathrm{g})$ internal surface iris of EHF half-cell; (h) internal surface iris of half-cell formed by spinning. White zones inside the KAM maps represent nonindexed points. 

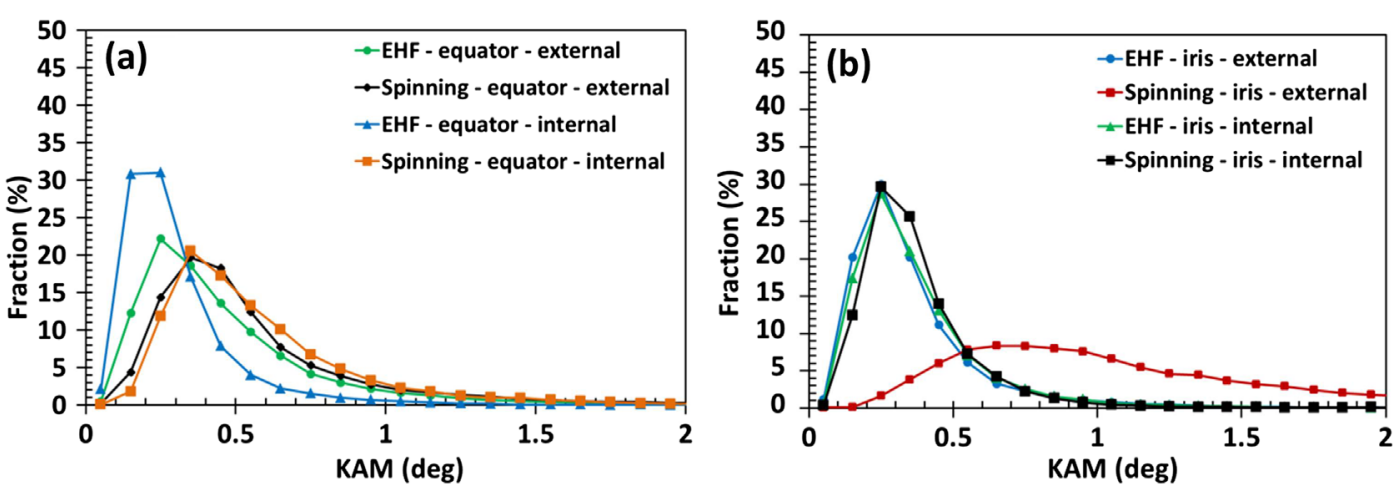

FIG. 18. Local misorientation distribution at the external and internal surfaces for specimens extracted from half-cells formed by EHF and spinning: (a) specimens obtained from equator; (b) specimens obtained from iris.

understand the difference of hardness found through thickness between the external and internal surfaces. The first $200 \mu \mathrm{m}$ of thickness close to the external surface showed a hardness of 92 (HV 0.2), while the $200 \mu \mathrm{m}$ close to the internal surface showed a hardness of 75 (HV 0.2). TEM specimens were extracted in the first 100-200 $\mu \mathrm{m}$ through thickness and close to the surfaces. The samples were prepared following a standard mechanical polishing procedure up to a thickness of $100 \mu \mathrm{m}$. They were then thinned down up to $30 \mu \mathrm{m}$ by dimpling and thinned further by ion milling.

The TEM used for imaging and analyses was a FEI Talos F200X operated in both STEM and TEM modes at $200 \mathrm{kV}$ accelerating voltage.

A bright field image of the sample extracted at the external surface is shown in Fig. 19(a). This zone shows extensive nanotwinning and the interaction of dislocations with nanotwins [white arrows in Fig. 19(a)]. A bright field image of a specimen extracted in the first $100 \mu \mathrm{m}$ close to the internal surface is shown in Fig. 19(b), where bundles of dislocations are present but no twins. This difference in deformation mechanism could explain the difference in hardness found between the external and internal surfaces for this specimen.
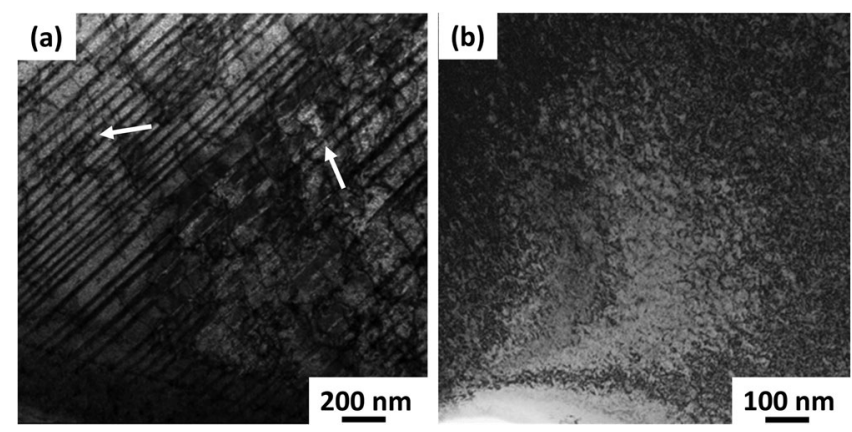

FIG. 19. Bright field TEM images of specimens extracted through thickness at the equator of the EHF niobium half-cell: (a) specimen obtained close to external surface; (b) specimen obtained close to internal surface.
In literature it was reported that for deformation at high strain rates, twinning might become predominant in bcc metals [14-34] and this seems to be confirmed for niobium.

\section{DISCUSSION}

The main aim of this paper is to present the procedure used to form SRF half-cells of niobium by electrohydraulic forming (EHF) and to compare the damage caused to the material by this high-speed forming technique with traditional forming techniques (i.e., spinning). Hardness measurements taken on the external and internal surfaces of SRF half-cells showed a higher hardness for spinning than for EHF along the half-cell profile. However, the two forming techniques showed the same hardness at the iris zone (results shown in Fig. 10). A similar result was found by measuring the hardness through thickness along the whole profile of the half-cell. Afterwards, RRR measurements were performed to assess the change of RRR caused by forming. It was found that the two techniques are causing the same level of RRR decrease along the radial direction of the half-cell, while a higher RRR drop was found in circumferential direction for EHF compared to spinning. However, the difference of RRR drop between the two techniques is still inside the standard deviation associated to the measurements. For this reason, it can be concluded that both forming techniques exhibit comparable drops of RRR which are acceptable for CERN specifications. In addition, a full recovery of the RRR value was obtained with a thermal treatment of $600{ }^{\circ} \mathrm{C}-5 \mathrm{~h}$. From microstructure investigations (hardness and grain size), this thermal treatment was proved to be enough to recover dislocations without causing any recrystallization of the material.

However, in order to investigate at microscale the damage level and distribution induced inside niobium by both EHF and spinning, EBSD analyses were performed.

From the KAM map extracted through the whole thickness (Fig. 15), in the sample extracted at the equator of the EHF half-cell, a gradient of damage was found with higher values at the external surface and very low values at the 

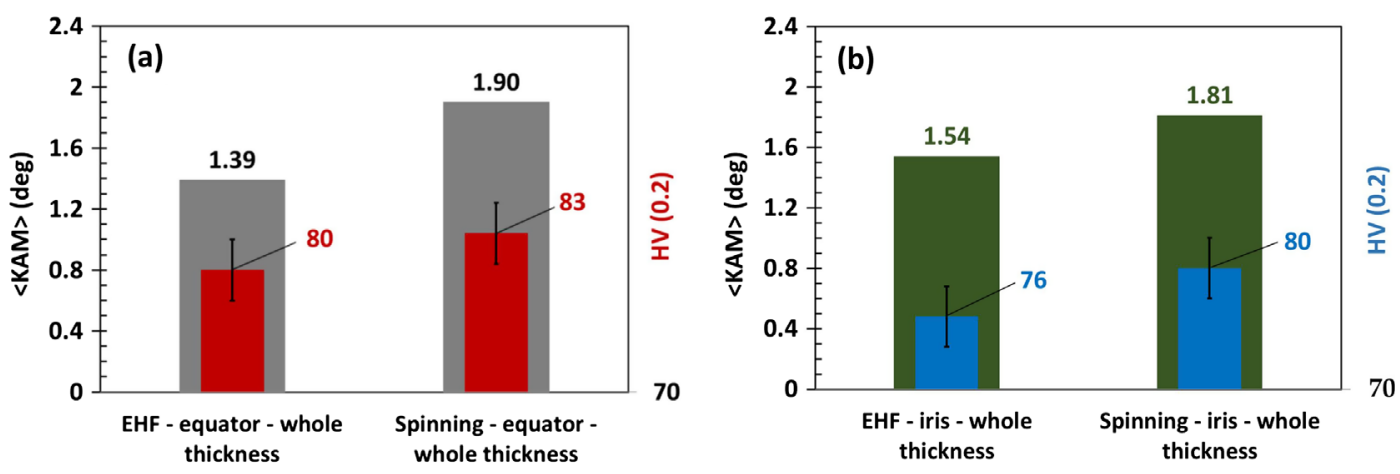

FIG. 20. Weighted average $\mathrm{KAM}(\langle\mathrm{KAM}\rangle)$ and average hardness values measured through the whole thickness: (a) specimens extracted from equator; (b) specimens extracted from iris.

internal surface ( $\mathrm{rf}$ surface). Instead, the distribution of damage through thickness appears uniform for the sample extracted at the equator for the half-cell formed by spinning and as well for both samples extracted close to the iris. In order to quantify the difference of damage between the two forming techniques, the weighted average KAM was calculated and plotted. The average values of hardness measured through thickness have been added in the same graph to further support the trend found for both measurements. The results are shown in Figs. 20(a) and 20(b). From Fig. 20(a) it can be seen that the weighted average KAM for the whole thickness is lower for EHF (1.39) than spinning (1.90). The same trend is found for average hardness values ( 80 for EHF and 83 for spinning), even if the standard deviation bars of the two measurements overlap. At the iris [Fig. 20(b)], the weighted average KAM is lower for EHF (1.54) compared to spinning (1.81). The same trend was found for hardness (76 for EHF and 80 for spinning). From these results, it can be concluded that EHF is not worsening the damage caused in the material, but instead it seems to have a lower damage through the whole thickness compared to spinning. When further EBSD analyses were performed in the first $200 \mu \mathrm{m}$ through thickness close to external and internal surfaces, it was noticed that at the equator, the forming causes the same damage for both techniques at the external surface while the damage at the internal surface is lower for EHF compared to spinning
[Fig. 21(a)]. The values of hardness through thickness in the first $200 \mu \mathrm{m}$ close to external and internal surfaces confirmed the same result. At the iris, a much lower damage was found at the external surface for EHF as shown by Fig. 21(b) where the difference between the two forming techniques is high both in terms of KAM and hardness through thickness. However, the damage caused at the internal surface of the iris is comparable for both forming techniques [KAM maps Figs. 17(g), 17(h), and 21(b)].

\section{CONCLUSIONS}

This paper has presented the electrohydro forming of SRF half-cells in niobium. The first part has shown the simulations which are necessary in order to optimize the forming procedure, while in the second part, this highspeed forming technique was compared with spinning in terms of microstructure of the formed niobium half-cell.

From the results obtained by microstructure analyses, it can be concluded that the level of damage caused inside the material is the same for both forming techniques or in some areas lower for EHF than for spinning. These first results are promising to proceed further with the production of niobium half-cells since EHF benefits from increase of metal formability, reduced springback, and preservation of surface roughness as well as a high reduction of forming time. Indeed, a half-cell can be formed in a few seconds
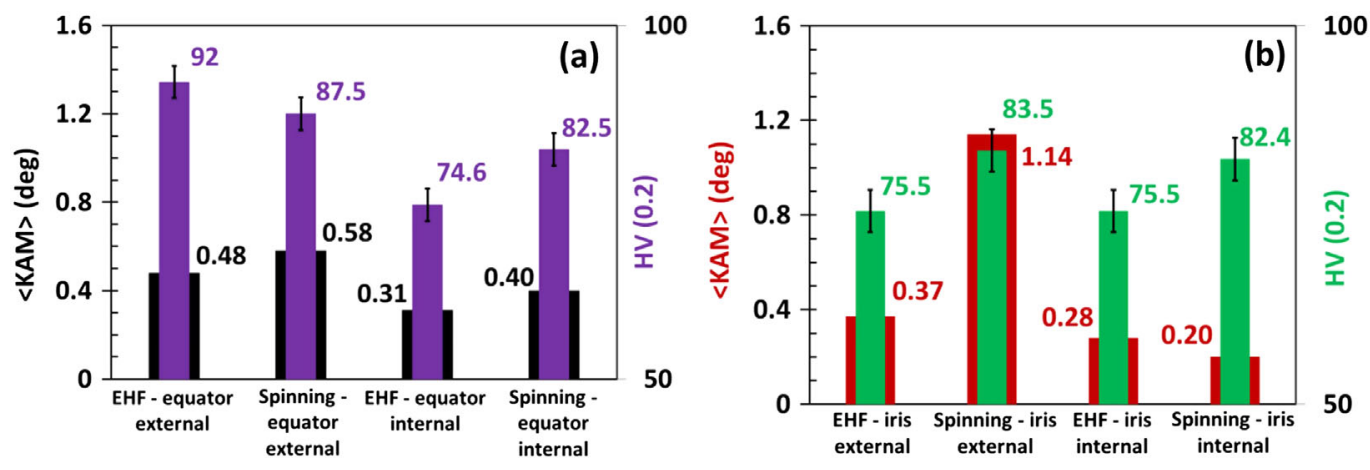

FIG. 21. Weighted average $\mathrm{KAM}(\langle\mathrm{KAM}\rangle)$ and average hardness values measured in the first $200 \mu \mathrm{m}$ close to the external and internal surfaces: (a) specimens extracted from equator; (b) specimens extracted from iris. 
versus the hours required for spinning. Further activities are ongoing to investigate EHF of large grains niobium.

\section{ACKNOWLEDGMENTS}

Dr. Alla Sologubenko and Dr. Karsten Kunze from the Scientific Center for Optical and Electron Microscopy (SCOPEM) at ETH Zürich are gratefully acknowledged for the TEM samples preparation and investigations.

[1] FCC Week 2016, https://indico.cern.ch/event/438866/.

[2] F. Gerigk, Conceptual Design of the Low-Power and HighPower SPL - A Superconducting H- Linac at CERN (CERN, Geneva, 2014).

[3] O. Capatina et al., Mechanical design considerations for $\beta=1$ cavities, in Proceedings of SRF2011, Joint Accelerator Conferences Website (JACoW), Chicago, 2011 (CERN, Geneva, 2011), paper THIOA06.

[4] I. Gonin, I. Jelezov, H. Kaiser, T. Khabibuline, W. Singer, and X. Singer, Hydroforming of back extruded niobium tubes, in Proceedings of the 1999 Workshop on RF Superconductivity, Santa Fe, NM, 1999 (CERN, Geneva, 1999), paper WEP 042.

[5] W. Singer, X. Singer, I. Jelezov, and P. Kneisel, Hydroforming of elliptical cavities, Phys. Rev. ST Accel. Beams 18, 022001 (2015).

[6] H. Padamsee, J. Knobloch, and T. Hays, $R F$ Superconductivity for Accelerators (Wiley-VCH, Weinheim, Germany, 2008).

[7] F. Marhauser, JLAB SRF Cavity Fabrication Errors, Consequences and Lessons learned, in Proceedings of IPAC2011, San Sebastian, Spain (EPS-AG, Spain, 2011), paper MOPC 115.

[8] S. Atieh et al., First results of SRF cavity fabrication by electro-hydraulic forming at CERN, in Proceedings of SRF2015, Whistler, British Columbia, Canada (2015), paper THAA05.

[9] C. C. Wong, T. A. Dean, and J. Lin, A review of spinning, shear forming and flow forming processes, Int. J. Machine Tools Manufacture 43, 1419 (2003).

[10] R. Smerd, S. Winkler, C. Salisbury, M. Worswick, D. Lloyd, and M. Finn, High strain rate tensile testing of automotive aluminum alloy sheet, Int. J. Impact Eng. 32, 541 (2005).

[11] M. Seth, V. Vohnout, and G. S. Daehn, Formability of steel sheet in high velocity impact, J. Mater Process Tech 168, 390 (2005).

[12] A. Melander, A. Delic, A. Bjorkblad, P. Juntunen, L. Samek, and L. Vadillo, Modelling of electro hydraulic free and die forming of sheet steels, Int. J. Mater. Form. 6, 223 (2013).

[13] Bmax commercial web site, http://www.bmax.com.

[14] G. T. Gray III, High-strain-rate deformation: Mechanical behavior and deformation substructures induced, Annu. Rev. Mater. Res. 42, 285 (2012).
[15] P. Follansbee and J. Weertman, On the question of flow stress at high strain rates controlled by dislocation viscous flow, Mech. Mater. 1, 345 (1982).

[16] G. R. Johnson and W. H. Cook, A Constitutive Model and Data for Metals subjected to large strains, high strain rates and high temperatures, in Proceedings of the 7th International Symposium on Ballistics, Netherlands (1983).

[17] F. J. Zerilli and R. W. Armstrong, Dislocation-mechanicsbased constitutive relations for material dynamics calculations, J. Appl. Phys. 61, 1816 (1987).

[18] A. C. Jeanson, G. Avrillaud, G. Mazars, F. Bay, E. Massoni et al., Identification du comportement mécanique dynamique de métaux par un essai d'expansion électromagnétique de tube, in CSMA 2013-11eme colloque national en calcul des structures, Giens, France, 2013.

[19] A. C. Jeanson, G. Taber, G. Daehn, G. Avrillaud, G. Mazars, F. Bay, N. Jacques, and M. Arrigoni, Identification of material constitutive parameters for dynamic applications: Magnetic Pulse Forming (MPF) and electrohydraulic forming (EHF), in The 6th International Conference on High Speed Forming (2014).

[20] A. C. Jeanson, F. Bay, N. Jacques, G. Avrillaud, M. Arrigoni, and G. Mazars, A coupled experimental/ numerical approach for the characterization of material behaviour at high strain-rate using electromagnetic tube expansion testing, Int. J. Impact Eng. 98, 75 (2016).

[21] P. L'Eplattenier, G. Cook, C. Ashcraft, M. Burger, J. Imbert, and M. Worswick, Introduction of an electromagnetism module in LS-DYNA for coupled mechanicalthermal-electromagnetic simulations, Steel Research Int. 80, 351 (2009).

[22] W. Singer, A. Ermakov, and X. Singer, TTC Report, 2010, http://flash.desy.de/reports_publications/tesla_reports/ttc_ reports_2010.

[23] W. Singer, Technical specification for large crystal niobium discs applied for the fabrication of $1.3 \mathrm{GHz}$ superconducting cavities, version B, DESY April 2006.

[24] P. Bauer and C. Antoine, Fermilab-Technical Division Note TD-06-008, 2006.

[25] C. A. Cooper, A. Wu, P. Bauer, and C. Antoine, Effect of Different Cutting Techniques on the Surface Morphology and Composition of Niobium, IEEE Trans. Appl. Supercond. 19, 1399 (2009).

[26] T. R. Bieler, N. T. Wright, F. Pourboghrat, C. Compton, K. T. Hartwig, D. Baars, A. Zamiri, S. Chandrasekaran, P. Darbandi, H. Jiang, E. Skoug, S. Balachandran, G. E. Ice, and W. Liu, Physical and mechanical metallurgy of high purity $\mathrm{Nb}$ for accelerator cavities, Phys. Rev. ST Accel. Beams 13, 031002 (2010).

[27] W. Singer, SRF cavity fabrication and materials, in Proceedings of the CAS-CERN Accelerator School: Superconductivity for Accelerators, Erice, Italy, 2013 edited by R. Bailey (CERN, Geneva, 2014).

[28] W. D. Callister, Jr., Materials Science and Engineering: An Introduction (John Wiley \& Sons, New York, 2007).

[29] E. J. Mittemeijer, Fundamentals of Materials Science (Springer-Verlag, Berlin Heidelberg, 2010). 
[30] S. I. Wright, M. M. Nowell, and D. P. Field, A review of strain analysis using electron backscattered diffraction, Microscopy Microanalysis 17, 316 (2011).

[31] F. Léaux, Ph.D. dissertation, Université Lille 1 Science et Technologies, 2012, https://ori-nuxeo.univ-lille1.fr/ nuxeo/site/esupversions/005e40aa-d0b5-4a87-b9e0b949ca00a05f.

[32] A. Romanenko and H. Padamsee, The role of near-surface dislocations in the high magnetic field performance of superconducting niobium cavities, Supercond. Sci. Technol. 23, 045008 (2010).

[33] C. Antoine, Materials and Surface Aspects in the Development of SRF Niobium Cavities, EUCARD series on Accelerator Science (EUCARD-BOO-2012-001) (2011).

[34] P. J. Ferreira, J. B. Vander Sande, M. Amaral Fortes, and A. Kyrolainen, Microstructure development during highvelocity deformation, Metall. Mater. Trans. A 35, 3091 (2004). 